\title{
Rickettsiae phylogeny: a multigenic approach
}

\author{
Correspondence \\ Líbia Zé-Zé \\ libia.zeze@insa.min-saude.pt
}

Received 11 August 2006

Revised 3 October 2006

Accepted 12 October 2006

\section{Liliana Vitorino, ${ }^{1}$ Ivo M. Chelo, ${ }^{1}$ Fatima Bacellar ${ }^{2}$ and Líbia Zé-Zé ${ }^{1} \dagger$}

\author{
${ }^{1}$ Universidade de Lisboa, Faculdade de Ciências. Centro de Genética e Biologia Molecular and \\ Instituto de Ciência Aplicada e Tecnologia. Edifício ICAT, Campus FCUL, Campo Grande, \\ 1749-016 Lisboa, Portugal \\ ${ }^{2}$ CEVDI, Instituto Nacional de Saúde Dr Ricardo Jorge, INSA, Av. Padre Cruz, 1649-016 \\ Lisboa, Portugal
}

\begin{abstract}
The development of molecular taxonomic methods has provided a large amount of data in the reorganization of Rickettsiae taxonomy. Nevertheless, phylogenetic relationships among some groups and species delimitation remain unclear. To clarify rickettsial phylogeny, a multigenic approach was used for the first time for the genus Rickettsia, based on simultaneous analyses of eight loci: atpA, recA, virB4, dnaA, dnaK, rrl-rrf internal transcribed spacer, ompA and gltA.

Concatenation of different nucleotide sequences resulted in an improvement in phylogenetic resolution when compared to single gene data. This multigenic approach has enabled the differentiation of many groups, including the spotted fever group which includes a great number of closely related species. The reliability of some previously recognized groups was evaluated.
\end{abstract}

\section{INTRODUCTION}

The genus Rickettsia belongs to the Alphaproteobacteria and represents a group of obligate intracellular endosymbionts of eukaryotic cells (Weiss \& Dasch, 1991). The genus subdivides into the conventionally well defined typhus group (TG) and spotted fever group (SFG), based mainly on phenotypic and serological features. The genus Rickettsia also includes several marginal species, such as Rickettsia canadensis and Rickettsia bellii, the most divergent species within the Rickettsiae (Stothard et al., 1994). The recently sequenced $R$. bellii genome was shown to be larger than the other rickettsial genomes sequenced so far and exhibits little colinearity with any of them, suggesting that this species may have retained several ancestral features lost in other lineages in the course of evolution (Ogata et al., 2006).

The TG includes Rickettsia prowazekii and Rickettsia typhi, which are the aetiological agents of insect-borne epidemic and endemic typhus, respectively. The SFG consists of several species, some of which are the causative agents of well known tick-borne diseases, such as Rocky Mountain spotted fever (Rickettsia rickettsii) and Mediterranean spotted fever (Rickettsia conorii) (Raoult \& Roux, 1997).

tPresent address: CEVDI, Instituto Nacional de Saúde Dr Ricardo Jorge, INSA, Av. Padre Cruz, 1649-016 Lisboa, Portugal.

Abbreviations: $d N / d S$, non-synonymous/synonymous substitutions distances ratio; ITS, internal transcribed spacer; ML, maximumlikelihood; MLST, multilocus sequence typing; MP, maximum-parsimony; NJ, neighbour-joining; SFG, spotted fever group; TG, typhus group.

A table of GenBank/EMBL/DDBJ accession numbers for the sequences reported in this paper is available as supplementary data with the online version of this paper.
Rickettsiae phylogeny has been addressed by sequence analyses of certain genes, mostly those belonging to the sca (surface cell antigen) family (Roux \& Raoult, 1995, 2000; Roux et al., 1997; Fournier et al., 1998; Sekeyova et al., 2001; Ngwamidiba et al., 2006), enabling the division of this genus into six groups represented by the species: Rickettsia rickettsii, Rickettsia massiliae, Rickettsia helvetica, Rickettsia akari, Rickettsia prowazekii and Rickettsia canadensis. Nevertheless, a robust and accurate phylogeny of this genus has not yet been accomplished and ambiguities remain in the phylogenetic position of some species. Moreover, for the last two decades, more novel Rickettsia species have been described, contributing to an increase in the complexity of rickettsial taxonomy.

As recommend by Stackebrandt et al. (2002), the use of several genes scattered across the bacterial genome boosts the informative level of phylogenetic data, improving the discriminatory power and the robustness of the phylogenetic relationships. Ideally, one should use data from housekeeping genes, which are ubiquitous within the population and tend to evolve at a moderate rate, and from hypervariable genes which might encode outer-surface proteins and typically evolve at an accelerated rate (Cooper \& Feil, 2004). Hence, we have analysed eight target nucleotide sequences, atpA, recA, virB4, dnaA, dnaK, rrl$r r f$ internal transcribed spacer (ITS), ompA and $g l t A$, selected as representative of different genomic functions believed to be active in most rickettsial species. In the present study, we compared the phylogenetic trees derived from different datasets and analysed for the first time the discriminatory capacity of concatenated sequences to infer stable phylogenetic relationships within rickettsial species. With this 
multigenic approach we have been able to achieve a good overall phylogenetic resolution at different levels of genetic resemblance.

\section{METHODS}

Bacterial strains, culture conditions and DNA extraction. Strains used are listed in Table 1 . The rickettsiae were propagated on Vero cell (E-6 clone) monolayers in Eagle's minimal medium (MEM; Gibco-BRL) supplemented with $1 \%(\mathrm{v} / \mathrm{v})$ glutamine and $8 \%(\mathrm{v} / \mathrm{v})$ fetal bovine serum at $32{ }^{\circ} \mathrm{C}$ for $5-6$ days. Infected cell

Table 1. Rickettsial strains used in this study

\begin{tabular}{|c|c|c|}
\hline Species & Strain & $\begin{array}{c}\text { Source/geographic } \\
\text { origin }\end{array}$ \\
\hline Rickettsia conorii & Malish $7^{\star}$ & Human/South Africa \\
\hline Rickettsia conorii & PoTiR12 & Vector/Portugal \\
\hline Rickettsia conorii & PoHuR1258 & Human/Portugal \\
\hline Rickettsia conorii & PoHuR4776 & Human/Portugal \\
\hline Israeli tick typhus & PoHuR4726 & Human/Portugal \\
\hline Israeli tick typhus & PoHuR8122 & Human/Portugal \\
\hline Israeli tick typhus & PoHuR1450 & Human/Portugal \\
\hline Israeli tick typhus & PoHuR8756 & Human/Portugal \\
\hline Israeli tick typhus & PoTiRB28 & Vector/Portugal \\
\hline $\begin{array}{l}\text { Rickettsia sibirica subsp. } \\
\text { monglotimonae }\end{array}$ & PoHuR10991 & Human/Portugal \\
\hline Rickettsia honei & TT-118† & Vector/Thailand \\
\hline Rickettsia slovaca & PoTiR24 & Vector/Portugal \\
\hline Rickettsia slovaca & PoTiR30 & Vector/Portugal \\
\hline Rickettsia slovaca & $13-\mathrm{B} \dagger$ & Vector/Slovakia \\
\hline Rickettsia massiliae & MTU1† & Vector/France \\
\hline Rickettsia sp. Bar29 & Bar29† & Vector/Spain \\
\hline Rickettsia aeschlimannii & $\mathrm{MC} 16 \dagger$ & Vector/Morocco \\
\hline Rickettsia montanensis & $\mathrm{M} / 56 \dagger$ & Vector/USA \\
\hline Rickettsia helvetica & $\mathrm{C9P9 \dagger}$ & Vector/Switzerland \\
\hline Rickettsia helvetica & PoTiR43 & Vector/Portugal \\
\hline Rickettsia sibirica & $246^{\star}$ & Vector/Siberia \\
\hline Rickettsia rhipicephali & $3-7-6 \dagger$ & Vector/USA \\
\hline Rickettsia akari & $\mathrm{MK} \dagger$ & Human/USA \\
\hline Rickettsia bellii & $369 \mathrm{~L} 42-1 \dagger$ & Vector/USA \\
\hline Rickettsia canadensis & $\# 2678 \dagger$ & Vector/Canada \\
\hline Rickettsia africae & EFS-5† & Vector/USA \\
\hline Rickettsia japonica & $\mathrm{YM} \dagger$ & Vector/Japan \\
\hline Rickettsia sp. A-167 & A- $167 \dagger$ & Vector/Russia \\
\hline Rickettsia australis & Philips $\dagger$ & Human/Australia \\
\hline Rickettsia sp. BJ-90 & BJ-90† & Vector/China \\
\hline Rickettsia prowazekii & Madrid $E^{\star}$ & Human/Spain \\
\hline Rickettsia typhi & Wilmington $\dagger$ & Human/USA \\
\hline Rickettsia rickettsii & R Bitterroot $\dagger$ & Vector/USA \\
\hline Rickettsia felis & URRWFXCal $\dagger$ & Vector/France \\
\hline
\end{tabular}

${ }^{\star}$ Genome sequence retrieved from GenBank database: AE006914, R. conorii; AABW00000000, R. sibirica; AJ235269, R. prowazekii. $†$ Strains generously provided by D. Raoult (Unité des Ricketsies, Faculté de Médecine, Université de la Méditerrannée, Marseille, France). All Portuguese isolates belong to the CEVDI collection. cultures were monitored for the presence of rickettsiae. Harvesting was done when the degree of infection was optimal, as determined by Gimenez staining (Gimenez, 1964). The cultures were frozen and unfrozen three times, and then centrifuged at $121 \mathrm{~g}$ for $30 \mathrm{~min}$. The supernatants were recovered, then the rickettsiae were harvested by $30 \mathrm{~min}$ centrifugation at $7740 \mathrm{~g}$ and resuspended in ultra-pure water. DNA was extracted using Qiagen columns according to the DNAeasy protocol for the Animal Tissue Kit.

PCR amplification and gene sequencing. Primers used to amplify and sequence the several gene targets are listed in Table 2. PCR was performed in a $50 \mu \mathrm{l}$ reaction mixture containing $1 \mathrm{pmol}$ each primer, $200 \mu \mathrm{M}$ each dATP, dGTP, dCTP and dTTP (Invitrogen), $1.75 \mathrm{U}$ Taq polymerase (Invitrogen), $2 \mathrm{mM} \mathrm{MgCl}_{2}$, $0.5 \times$ BSA, $1 \times$ Taq buffer and 50-100 ng genomic DNA. Amplification was carried out in a DNA thermocycler (TGradient; Biometra) using the following conditions: $5 \mathrm{~min}$ of initial denaturation at $96^{\circ} \mathrm{C}$, then 35 cycles at $94^{\circ} \mathrm{C}$ for $1 \mathrm{~min}, 52^{\circ} \mathrm{C}$ for $1 \mathrm{~min}$ and $72^{\circ} \mathrm{C}$ for $1 \mathrm{~min}$. The amplification was completed by holding for 5 min at $72{ }^{\circ} \mathrm{C}$ to allow complete extension of the PCR product. When no amplification product was obtained, the annealing temperature was lowered to $50{ }^{\circ} \mathrm{C}$ and the $\mathrm{MgCl}_{2}$ concentration was raised to $2.5 \mathrm{mM}$. PCR products were visualized by ethidium bromide staining after electrophoresis in a $1 \%(\mathrm{w} / \mathrm{v})$ agarose gel and their sizes were estimated by comparison with a molecular mass standard ( $1 \mathrm{~kb}$ plus DNA ladder; Gibco-BRL). The PCR products were purified using the Jet Quick-PCR Purification Kit (Genomed) as described by the manufacturer. The purified PCR products were sequenced in both directions using an automated DNA capillary sequencer CEQ 2000-XL (Beckman Coulter) and a dye-labelled dideoxy termination method (DTCS, Dye Terminator Cycle Sequencer Start Kit; Beckman Coulter). Sequences were proofread, edited and merged into composite sequences using CEQ 8000 software (Beckman Coulter). Accession numbers of nucleotide sequences are listed in supplementary Table S1 (available with the online version of this paper). All sequence data were posted at the multilocus sequence typing (MLST) website, http://rickettsia.mlst.net (Aanensen \& Spratt, 2005).

Data analysis. For phylogenetic inference, nine different alignments were created, eight corresponding to the alignment of sequences of each gene: atpA, recA, dnaA, rrl-rrf ITS, virB4, gltA, $d n a K$ and ompA and one alignment corresponding to the concatenation of all gene sequences. All alignments were made using the CLUSTAL W program (Thompson et al., 1997). For protein-encoding genes, DNA alignments were made based on the corresponding amino acid alignments by using CodonAlign 2.0 (Hall, 2004). Aligned regions with gaps were removed from the alignment before phylogenetic analysis.

Phylogenetic analysis including neighbour-joining (NJ), maximumparsimony (MP) and maximum-likelihood (ML) trees of DNA sequence alignments were conducted using PAUP ${ }^{*} 4.0 \mathrm{~b} 10$ software (Swofford, 2000). Genetic distances used in NJ trees are Kimura twoparameter model distances with a transition : transversion ratio of $2: 1$. Non-synonymous/synonymous substitutions distances $(\mathrm{d} N / \mathrm{d} S)$ ratios, as described by Nei \& Gojobori (1986), were calculated using MEGA3 software (Kumar et al., 2004). Analysis of protein sequences were done using several programs present in the PHYLIP software package, version 3.6 (Felsenstein, 1989). Bootstrap analysis was made with 1000 replicates except in ML where only 100 replicates were generated. For the Bayesian approach, MrBayes software version 3.0b4 (Huelsenbeck \& Ronquist, 2001) was used. Each analysis consisted of $2.0 \times 10^{7}$ generations from a random starting tree and four Markov chains (with default heating values) sampled every 100 generations. The first 8000 sampled trees were discarded resulting in a set of 12000 analysed trees sampled after stationarity. To prevent reaching only apparent 
Table 2. Primers used for PCR amplification and sequencing

\begin{tabular}{|c|c|c|c|c|c|c|c|}
\hline Gene & Description & Primer sequence $\left(5^{\prime}-3^{\prime}\right)^{*}$ & $\begin{array}{l}\text { Amplicon } \\
\text { size (nt) }\end{array}$ & $\begin{array}{c}\text { No. of } \\
\text { nucleotides in } \\
\text { phylogenies }\end{array}$ & $\begin{array}{l}\mathrm{dN} / \mathrm{d} S \\
\text { ratio } \dagger\end{array}$ & $\begin{array}{l}\text { SFG mean } \\
\text { distance }\end{array}$ & Reference \\
\hline $\operatorname{atp} A$ & ATP synthase F1 $\alpha$ subunit & $\begin{array}{l}\text { Fw: AGTACAGACATATCGAGATGA } \\
\text { Rv: CGACTTACCGAAATACCGAC }\end{array}$ & 745 & 600 & 0.050 & 0.022 & This study \\
\hline $\operatorname{rec} A$ & RecA recombination protein & $\begin{array}{l}\text { Fw: CTCACTTGGGCTAGATATAGC } \\
\text { Rv: CTTCTTTATCTTTAATGGAGCC }\end{array}$ & 578 & 482 & 0.039 & 0.027 & This study \\
\hline $\operatorname{virB4}$ & Virulence protein B4 & $\begin{array}{l}\text { Fw: TCTATAGTACATGATTCTGCT } \\
\text { Rv: TGATTACCGAGTGTAGTATTATG }\end{array}$ & 840 & 717 & 0.408 & 0.018 & This study \\
\hline$d n a K$ & Heat-shock protein 70 & $\begin{array}{l}\text { Fw: GCATTCTAGTCATACCGCC } \\
\text { Rv: CAAAAAATGAAAGAAACTGCTGA }\end{array}$ & 650 & 515 & 0.097 & 0.024 & This study \\
\hline$d n a A$ & $\begin{array}{l}\text { DnaA chromosomal } \\
\text { replication initiation protein }\end{array}$ & Fw: CTTTACAATCATTACGGTG & 788 & 597 & 0.111 & 0.021 & This study \\
\hline$r r l-r r f$ ITS & ITS & $\begin{array}{l}\text { Rv: GCAACTAAGCCCCATCC } \\
\text { Fw: GATAGGTCGGGTGTGGAAG }\end{array}$ & 350 & $287-323$ & NA & 0.057 & $\begin{array}{l}\text { Vitorino } \\
\text { et al. (2003) }\end{array}$ \\
\hline ompA & Outer-membrane protein & $\begin{array}{l}\text { Rv: TCGGGATGGGATCGTGTG } \\
\text { Fw: TGGCGAATATTTCTCCAAAA }\end{array}$ & 532 & 450 & 1.905 & 0.057 & $\begin{array}{l}\text { Regnery } \\
\text { et al. (1991) }\end{array}$ \\
\hline gltA & Citrate synthase protein & $\begin{array}{l}\text { Rv: AGTGCAGCATTCGCTCCCCCT } \\
\text { Fw: GGGGGCCTGCTCACGGGCGG }\end{array}$ & 382 & 380 & 0.112 & 0.023 & $\begin{array}{l}\text { Roux et al. } \\
(1997)\end{array}$ \\
\hline & & Rv: ATTGCAAAAAGTACAGTGAAC & & & & & \\
\hline
\end{tabular}

${ }^{\star} \mathrm{Fw}$, Forward primer; Rv, reverse primer.

$\dagger$ NA, Not applicable.

stationarity, two separate runs were made for each analysis. Hierarchical likelihood ratio tests were conducted using a batch file supplied with MODELTEST 3.06 (Posada \& Crandall, 1998) to provide the evolutionary models used in ML and Bayesian analysis. Based on the work by Stothard et al. (1994), R. bellii was used as outgroup.

\section{RESULTS AND DISCUSSION}

Phylogenetic relationships inferred from throughout the genome are viewed as accurate estimates of those that would be obtained from an analysis of the entire genome, since the effects of location-dependent processes in sequence evolution can be reduced by sampling sites from different regions (Cummings et al., 1995). Hence, several target nucleotide sequences were selected as representative of different genomic functions (Table 2) believed to have an important role in the rickettsial life cycle, and with regard to their scattered distribution in the rickettsial chromosome (Andersson et al., 1998; Ogata et al., 2001). With this approach we expected to use sequences that were not contiguous in the rickettsial genome to minimize the effect of location-dependent nucleotide evolution (Cummings et al., 1995). Moreover, we used both housekeeping and hypervariable genes to provide a framework for studying closely related strains as well as different species (Cooper \& Feil, 2004). Although our main goal was not rickettsial strain characterization, considering that this data can be used in an MLST scheme, all sequence data were posted to an MLST database as a focal point for future storage and retrieval of rickettsial sequence, strain and host data.

Thirty-four rickettsial strains were analysed. For R. bellii and R. canadensis the PCR amplification of some of the genes was difficult or even impossible (supplementary Table S1, available with the online version of this paper). This was not unexpected, since a greater genomic divergence is recognized for these two species, preventing the amplification of some genes even when carried out with consensus primers (designed from known Rickettsia spp. sequences), as described by Sekeyova et al. (2001).

Due to the genetic closeness of rickettsial species, nucleotide sequences were used for phylogenetic analysis instead of protein sequences. Phylogenetic trees based on amino acid sequences were drawn at a preliminary stage (results not shown), but their lack of resolution was obvious even for the most divergent gene (ompA).

Given that the aim of this study was to conduct a wideranging clarification of the phylogenetic relationships within the genus Rickettsia by the use of different genes, we decided to make a joint analysis based on the assembly of all the sequences, to guarantee improvement of phylogenetic tree robustness. Incongruence length difference tests have been used for data concatenation; these tests can be good indicators of different evolutionary histories and are used to 
prevent the combination of incongruent data (Cunningham, 1997; Bull et al., 2003). To assess the adequacy of the all-gene tree to explain the evolution of each locus, we also performed Shimodaira-Hasegawa tests with re-sampling estimated log likelihood optimization (Goldman et al., 2000). In this test, different tree topologies were compared and their adequacy was tested based upon the comparison of their log likelihood values. Usually a $P$ value $\leqslant 0.05$ is used for the rejection of a given tree topology representing a hypothesis that might explain the data. Beyond the topology given by the all-data tree, the set of tested trees included the ML tree of each locus. ShimodairaHasegawa tests indicated that the tree constructed from concatenated data provides a plausible explanation for the evolutionary history of every gene (data not shown). Furthermore, single-gene trees were also obtained, enabling comparison of both approaches.

The analysis of the concatenated gene set (Fig. 1) was made using a Bayesian approach where cluster confidence is given by posterior probabilities (Huelsenbeck et al., 2001).

To account for different gene-specific evolutionary rates and rate patterns, the data were partitioned for the several genes, and an appropriate evolutionary model was used for each gene (Castoe et al., 2004). In addition, the confidence of the groups obtained by this approach was also evaluated by bootstrap values taken from ML trees. However, in the latter case no partition of the data was made and a single model of DNA evolution was computed for the entire set. While the chosen model for the ITS region and dnaK was HKY (Hasegawa et al., 1985) with gamma variation, for all the others the GTR model (Lanave et al., 1984), also with gamma variation, was selected. In $\operatorname{atp} A$, a proportion of invariable sites were considered. In the ML analysis, the GTR + G + I model was used for the entire set.

The analysis of the all-gene phylogenetic tree (Fig. 1) allows the recognition of several rickettsial groups whose phylogenetic clustering seems to be robust, with ML bootstrap values above $70 \%$ and posterior probability values close to 1.00. Most of these groups were also recovered in MP and $\mathrm{NJ}$ trees (results not shown), indicating that the increase in tree resolution can be attributed to an increase in phylogenetic signal and not to the reconstruction method used. Moreover, these results are compatible with singlegene phylogenies (Fig. 2), since there are no mutually exclusive groups with significant bootstrap support. In Fig. 2, NJ trees of single-gene phylogenies are shown. Bootstrap proportions are given only for the NJ method, but
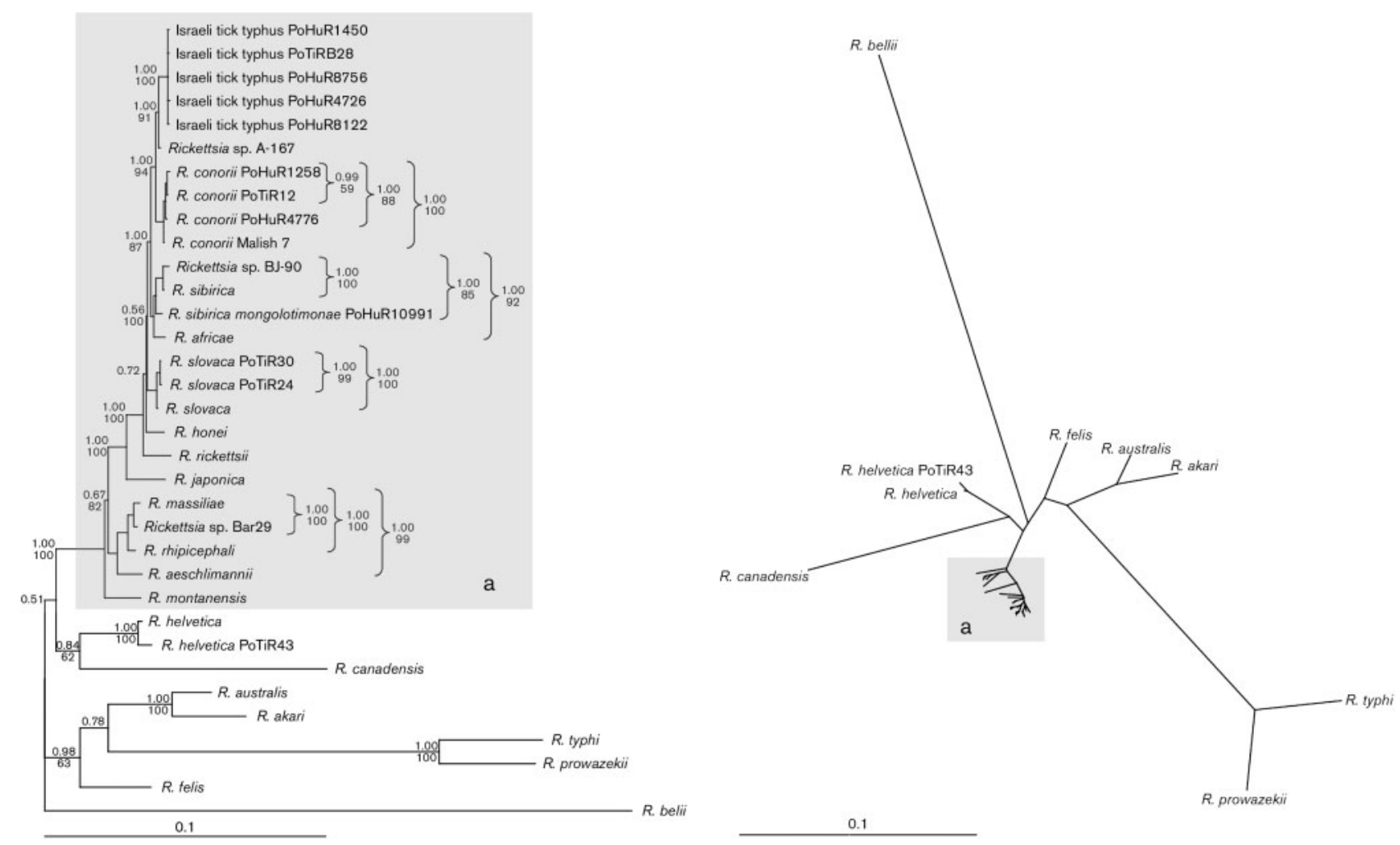

Fig. 1. MrBayes tree using concatenated sequences: atp $A, \operatorname{rec} A, d n a A, r r l-r r f$ ITS, virB4, gltA, dnaK and ompA. The radial tree layout is shown to give a better visualization of the overall Rickettsiae phylogeny, and the phylogram is shown to highlight the lineage diversity within the SFG ( $\mathrm{a}$, in grey). In the phylogram posterior probabilities and ML bootstrap values are indicated to provide branch support. Bar, $10 \%$ sequence divergence. 

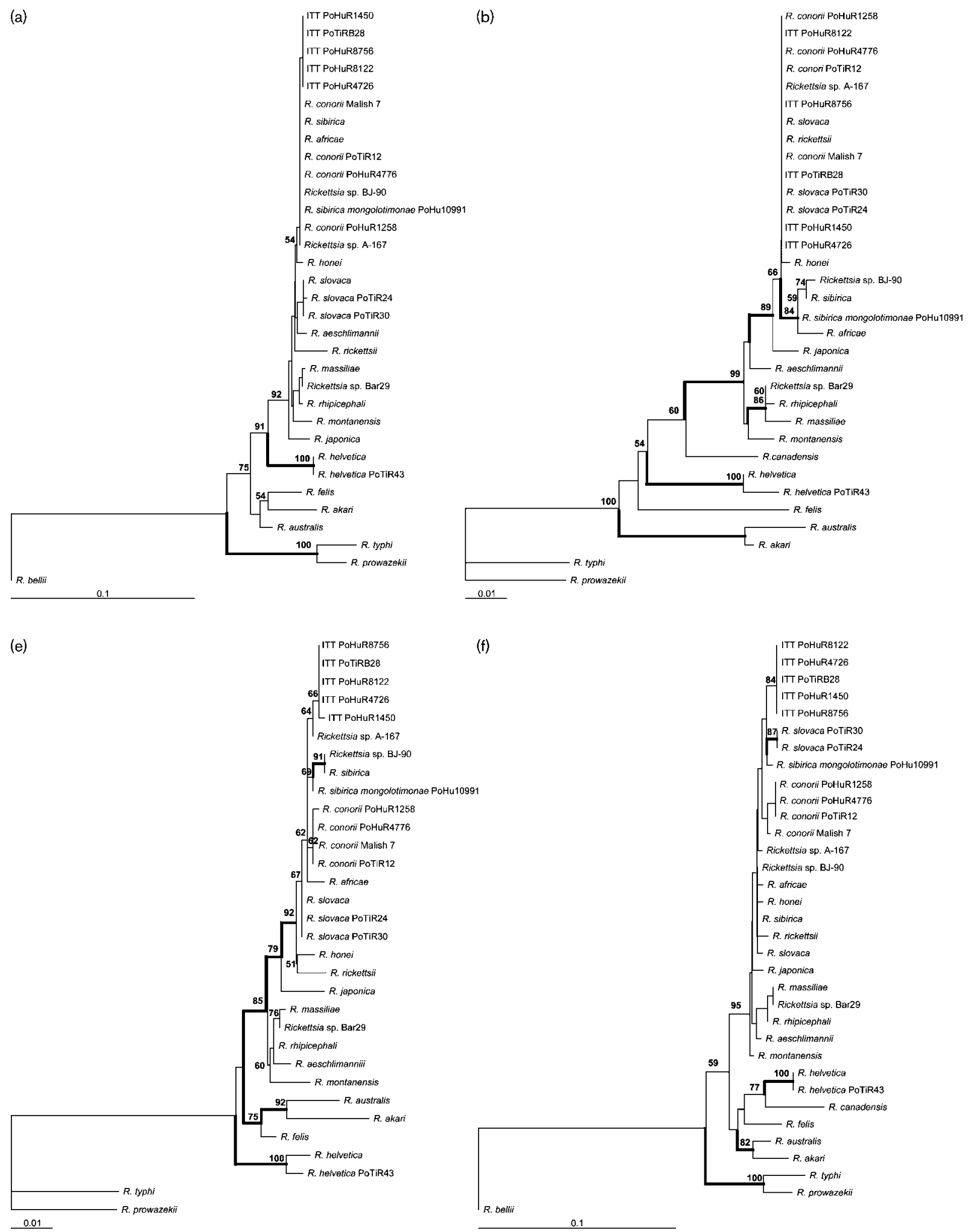

Fig. 2. For legend see opposite. 

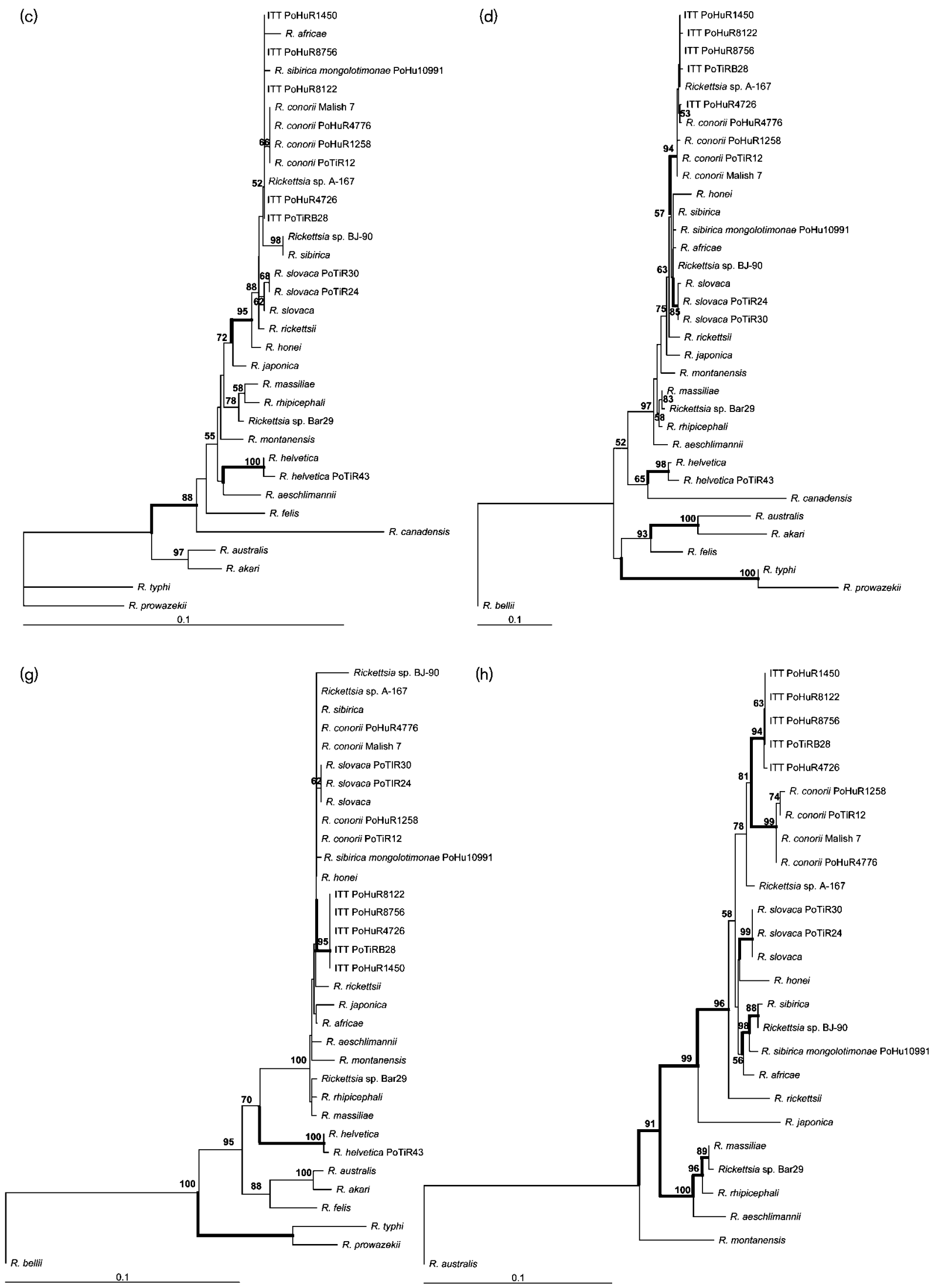

Fig. 2. $\mathrm{NJ}$ tree obtained from (a) atpA, (b) recA, (c) dnaA, (d) rrl-rrf ITS, (e) virB4, (f) gltA, (g) dnaK and (h) ompA sequences, and Kimura two-parameter model distances. Bootstrap proportions are given only for the NJ method. Only bootstrap values $>70 \%$ are shown. Branches leading to groups also supported by MP and ML trees are highlighted. 
branches leading to groups also supported by MP and ML trees, with bootstrap values $>70 \%$, are highlighted.

The phylogeny obtained with all the data also has the advantage of containing information from genes with different rates of evolution that are informative at different levels. This is particularly important since the phylogenetic inferences to be made are aimed both at the comparison of genetically close taxa of the traditional SFG and of the Rickettsia genus as a whole. ompA and the ITS region, which in the SFG have a mean genetic distance (Kimura twoparameter distance with a transition:transversion ratio $2: 1)$ of 0.057 nucleotide substitutions per site, are suitable for the clarification of phylogenetic relationships within the SFG. In contrast, phylogenies obtained from atpA, $\operatorname{rec} A$, dnaA, virB, gltA and $d n a K$, in which the mean intra-SFG group distances vary between 0.018 and 0.027 , are more suited to the establishment of more basal relationships, providing good delineation of the main groups of the Rickettsiae. As an example, one can see that phylogenies obtained from recA, virB and dnaK (Fig. 2) give strong support to most of the groups suggested by Sekeyova et al. (2001) and reviewed by Parola et al. (2005).

It is generally recognized that the phylogenetic relationships of the Rickettsiae are characterized by the presence of several groups with one or a few identifiable individuals, and that one group, the SFG, contains most of the recognized species. It is interesting to see that all of the groups recognized so far (Sekeyova et al., 2001) join each other in the middle of the phylogenetic tree, being connected by relatively short internal branches. Not surprisingly, the confidence of the clustering of any of these groups is hampered by low support in either bootstrap or posterior probability values. It should also be noted that these results do not support a monophyletic nature for the SFG. Both concatenated and single-gene sequence data indicate instead that SFG is divided into two groups: a monophyletic cluster, which includes the $R$. rickettsii group, the $R$. massiliae group (as defined by Sekeyova et al., 2001) and Rickettsia montana, and a second group, consisting of Rickettsia australis, Rickettsia akari and Rickettsia felis. The only exception to this evolutionary history is presented by the virB4 phylogeny, in which the SFG is seen as monophyletic, but without support. Regarding the $R$. akari group, only $R$. australis and $R$. akari form a well supported monophyletic cluster. This is to be expected, taking into consideration the hypothetical early separation of $R$. felis (as suggested in virB4, ITS and gltA phylogenies; Fig. $2 \mathrm{~d}$, e and g). As can be clearly seen from the radial tree in Fig. 1, R. felis branches from a central position in Rickettsia phylogeny. This could explain the variable phylogenetic positions or support values for $R$. felis in our study and also in other studies using different genes (Fournier et al., 2003; Zhu et al., 2005; Ngwamidiba et al., 2006). This relatedness between both the SFG and TG rickettsiae could account for the presence in the $R$. felis genome of genes exclusively shared with $R$. typhi; this may be the reason for their serological cross-reactivity, as pointed out by Ogata et al. (2005).

The previously mentioned monophyletic subgroup of the SFG rickettsiae is distinguished by the presence of many genetically close isolates. An overall good phylogenetic resolution is nevertheless possible, with many increasingly specific subgroups being recognized. In the $R$. rickettsii group, as mentioned by Sekeyova et al. (2001) the R. conorii complex is well established and can be readily subdivided into a group containing $R$. conorii Malish-like isolates, a group of Israeli-tick-typhus-like isolates and Rickettsia sp. A-167, the latter two being most closely related. This is in agreement with the resolution of this group as presented by Zhu et al. (2005). Although in this study, only Portuguese Israeli tick typhus isolates were used, ompA phylogenies including Israeli tick typhus strain ISTT (accession no. U83441) resulted in the same clear separation within the $R$. conorii complex (results not shown).

Another well defined monophyletic group includes not only Rickettsia sibirica strains, but also Rickettsia africae. Although the separation of these two can be made based on the all-gene phylogeny (most probably as a result of the information present in the ompA gene; see Fig. 2f), the proximity of $R$. africae to the $R$. sibirica group is also clear and becomes almost indistinguishable in recA phylogeny (Fig. 2b). The monophyly of this clade has already been recognized and it also contains Rickettsia parkeri and Rickettsia sp. strain S (Roux et al., 1997; Fournier et al., 1998; Roux \& Raoult, 2000; Sekeyova et al., 2001), which were not included in this study. Since evidence points towards it being a sister taxon to R. conorii, the attribution of a taxonomically defined rank such as species should be subject to further discussion.

In the remainder of the $R$. rickettsii group there is a distinct cluster with several Rickettsia slovaca strains (including two Portuguese isolates) and, of special notice, is the consistent position of Rickettsia japonica as the most divergent line in the group.

As found in the analysis of Sekeyova et al. (2001), the $R$. massiliae group, consisting of $R$. massiliae, Rickettsia sp. Bar29, Rickettsia rhipicephali and Rickettsia aeschlimannii is well supported, although only in the ompA single-gene tree (Fig. 2f) is it recovered with high bootstrap values by all methods. As shown in Fig. 1 the phylogenetic relationship between the $R$. massiliae group, the $R$. rickettsii group and Rickettsia montanensis is uncertain in this analysis. This fact, which can be seen as a difficulty in the resolution of the basal lines with regard to this diversification, gives credit to the previously recognized (Sekeyova et al., 2001) variable position of $R$. montanensis, thus care should be taken when considering its inclusion in the R. massiliae group, as suggested recently (Parola et al., 2005).

We should also draw attention to the phylogenetic position of $R$. canadensis, which is one of the most variable. In the 
dnaA gene phylogeny $R$. canadensis is even hypothesized as a member of the SFG, with high bootstrap proportions and posterior probability values. Nevertheless, this phylogeny is unique because of the long phylogenetic line leading to $R$. canadensis, most probably a result of an unusually high genetic divergence of this species. Indeed, $R$. canadensis was initially considered as a member of the TG on the basis of antigenic similarities; however, phylogenetic analysis (Roux et al., 1997; Fournier et al., 1998) has confirmed that this species constitutes a separate group from the SFG and TG rickettsiae. Remarkably, in spite of its unclear phylogenetic position, this species seems to be closer to $R$. helvetica than to members of the TG in the concatenated data analysis (Fig. 1).

As in the TG group, $R$. bellii is phylogenetically distant from all the other members of the Rickettsiae, which is in agreement with its distinct genomic (Ogata et al., 2006) and phenotypic (Philip et al., 1983) characteristics. Indeed, $R$. bellii is believed to be the only species of the first divergent line of the genus Rickettsia (Stothard et al., 1994). Despite its unquestionable use as an outgroup for other rickettsial species, it is impossible to recognize the order of divergence of the major groups due to the short length of internal branches, as already mentioned. This issue might be overemphasized by the missing data not only for $R$. bellii but also for $R$. canadensis. In fact, even if four gene sequences were obtained for these two species, only two of them would be common to both. This could seriously undermine inferences resulting from the presence of data from both species that would contribute to the clarification of the relatedness of the major rickettsial groups.

In conclusion, the combined use of the sequences of several housekeeping genes has led to the construction of a multi-gene phylogeny, which helps in clarifying the taxonomic structure of some groups of interest within the genus Rickettsia, particularly within the SFG. This group is not monophyletic, highlighting the limitations of serotyping in the delineation of taxonomic groups. Our study supports the distinctiveness of $R$. montanensis, which should thus be considered separate from the $R$. massiliae and $R$. rickettsii groups. In this analysis we have used several Portuguese isolates, which enabled us to take intraspecies variability into account. However, little resolution could be achieved at this level of relatedness. Obviously the multigenic approach to Rickettsiae phylogeny would be reinforced with the addition of more rickettsial species and new isolates that are constantly being described.

\section{ACKNOWLEDGEMENTS}

The ICAT/FCUL research team involved in this work strongly acknowledges its scientific leader, Professor Rogério Tenreiro, for general scientific supervision and engagement in providing the necessary resources and facilities. We also thank Edward J. Feil for helpful discussion of the final version of this manuscript. This publication made use of the Multi Locus Sequence Typing website at Imperial College London developed by David Aanensen and Man-Suen
Chan, and funded by the Wellcome Trust. L. Vitorino and I. M. Chelo are recipients of FCT research grants SFRH/BD/10676/2002 and SFRH/ $\mathrm{BD} / 10675 / 2002$, respectively.

\section{REFERENCES}

Aanensen, D. M. \& Spratt, B. G. (2005). The multilocus sequence typing network: mlst.net. Nucleic Acids Res 33 (Web Server issue), W728-W733.

Andersson, S. G. E., Zomorodipour, A., Andersson, J. O., SicheritzPontén, T., Alsmark, U. C. M., Podowski, R. M., Näslund, A. K., Eriksson, A.-S., Winkler, H. H. \& Kurland, C. G. (1998). The genome sequence of Rickettsia prowazekii and the origin of mitochondria. Nature 396, 133-140.

Bull, J. J., Huelsenbeck, J. P., Cunningham, C. W., Swofford, D. L. \& Waddell, P. J. (2003). Partitioning and combining data in phylogenetic analysis. Syst Biol 42, 384-397.

Castoe, T. A., Doan, T. M. \& Parkinson, C. L. (2004). Data partitions and complex models in Bayesian analysis: the phylogeny of gymnophthalmid lizards. Syst Biol 53, 448-469.

Cooper, J. E. \& Feil, E. J. (2004). Multilocus sequence typing - what is resolved? Trends Microbiol 12, 373-377.

Cummings, M. P., Otto, S. P. \& Wakeley, J. (1995). Sampling properties of DNA sequence data in phylogenetic analysis. Mol Biol Evol 12, 814-822.

Cunningham, C. W. (1997). Can three incongruence tests predict when data should be combined? Mol Biol Evol 14, 733-740.

Felsenstein, J. (1989). PHYLIP - Phylogeny Inference Package (Version 3.2). Cladistics 5, 164-166.

Fournier, P. E., Roux, V. \& Raoult, D. (1998). Phylogenetic analysis of the spotted fever Rickettsiae by study of the outer surface protein rOmpA. Int J Syst Bacteriol 48, 839-849.

Fournier, P. E., Dumler, J. S., Greub, G., Zhang, J., Wu, Y. \& Raoult, D. (2003). Gene sequence-based criteria for identification of new Rickettsia isolates and description of Rickettsia heilongjiangensis sp. nov. J Clin Microb 41, 5456-5465.

Gimenez, D. F. (1964). Staining Rickettsiae in yolk-sac cultures. Stain Technol 39, 135-140.

Goldman, N., Anderson, J. P. \& Rodrigo, A. G. (2000). Likelihoodbased tests of topologies in phylogenetics. Syst Biol 49, 652-670.

Hall, B. G. (2004). Phylogenetic Trees Made Easy: a How-To Manual for Molecular Biologists, 2nd edn. Sunderland, MA: Sinauer Associates.

Hasegawa, M., Kishino, H. \& Yano, T. (1985). Dating the humanape splitting by a molecular clock of mitochondrial DNA. J Mol Evol 22, 160-174.

Huelsenbeck, J. P. \& Ronquist, F. R. (2001). MrBayes: Bayesian inference of phylogenetic trees. Bioinformatics 17, 754-755.

Huelsenbeck, J. P., Ronquist, F., Nielsen, R. \& Bollback, J. P. (2001). Bayesian inference of phylogeny and its impact on evolutionary biology. Science 294, 2310-2314.

Kumar, S., Tamura, K. \& Nei, M. (2004). MEGA3: integrated software for Molecular Evolutionary Genetic Analysis and sequence alignment. Brief Bioinform 5, 150-163.

Lanave, C., Preparata, G., Saccone, C. \& Serio, G. (1984). A new method for calculating evolutionary substitution rates. J Mol Evol 20, 86-93.

Nei, M. \& Gojobori, T. (1986). Simple methods for estimating the number of synonymous and nonsynonymous nucleotide substitutions. Mol Biol Evol 3, 418-426. 
Ngwamidiba, M., Blanc, G., Raoult, D. \& Fournier, P.-E. (2006). ScaI, a previously undescribed paralog from autotransporter proteinencoding genes in Rickettsia species. BMC Microbiol 20, 6-12.

Ogata, H., Audic, S., Renesto-Audiffren, P., Fournier, P.-E., Barbe, V., Samson, D., Roux, V., Cossart, P., Weissenbach, J. \& other authors (2001). Mechanisms of evolution in Rickettsia conorii and $R$. prowazekii. Science 293, 2093-2098.

Ogata, H., Renesto, P., Audic, S., Robert, C., Blanc, G., Fournier, P. E., Parinello, H., Claverie, J. M. \& Raoult, D. (2005). The genome sequence of Rickettsia felis identifies the first putative conjugative plasmid in an obligate intracellular parasite. PLoS Biol 3, e248.

Ogata, H., La Scola, B., Audic, S., Renesto, P., Blanc, G., Robert, C., Fournier, P. E., Claverie, J. M. \& Raoult, D. (2006). Genome sequence of Rickettsia bellii illuminates the role of Amoebae in gene exchanges between intracellular pathogens. PLoS Genetics 2, 733-744.

Philip, R. N., Casper, E. A., Anacker, R. L., Cory, J., Hayes, S. F., Burgdorfer, W. \& Yunker, C. E. (1983). Rickettsia bellii sp. nov., a tick-borne rickettsia, widely distributed in the United States, that is distinct from the spotted fever and typhus biogroups. Int J Syst Bacteriol 33, 94-106.

Parola, P., Paddock, C. D. \& Raoult, D. (2005). Tick-borne rickettsioses around the world: emerging diseases challenging old concepts. Clin Microbiol Rev 18, 719-756.

Posada, D. \& Crandall, K. A. (1998). MODELTEST: testing the model of DNA substitution. Bioinformatics 14, 817-818.

Raoult, D. \& Roux, V. (1997). Rickettsioses as paradigms of new or emerging infectious diseases. Clin Microbiol Rev 10, 694-719.

Regnery, R. L., Spruill, C. L. \& Plikaytis, B. D. (1991). Genotypic identification of Rickettsiae and estimation of interspecies sequence divergence for portions of two rickettsial genes. J Bacteriol 173, $1576-1589$

Roux, V. \& Raoult, D. (1995). Phylogenetic analysis of the genus Rickettsia by $16 \mathrm{~S}$ rDNA sequencing. Res Microbiol 146, 385-396.

Roux, V. \& Raoult, D. (2000). Phylogenetic analysis of members of the genus Rickettsia using the gene encoding the outer-membrane protein $\mathrm{rOmpB}(o m p B)$. Int J Syst Evol Microbiol 50, 1449-1455.
Roux, V., Rydkina, E., Eremeeva, M. \& Raoult, D. (1997). Citrate synthase gene comparison, a new tool for phylogenetic analysis and its application for the Rickettsiae. Int J Syst Bacteriol 47, 252-262.

Sekeyova, Z., Roux, V. \& Raoult, D. (2001). Phylogeny of Rickettsia spp. inferred by comparing sequences of 'gene D'. which encodes an intracytoplasmic protein. Int J Syst Evol Microbiol 51, 1353-1360.

Stackebrandt, E., Frederiksen, W., Garrity, G. M., Grimont, P. A. D., Kämpfer, P., Maiden, M. C. J., Nesme, X., Rosselló-Mora, R., Swings, J. \& other authors (2002). Report of the ad hoc committee for the re-evaluation of the species definition in bacteriology. Int J Syst Evol Microbiol 52, 1043-1047.

Stothard, D. R., Clark, J. B. \& Fuerst, P. A. (1994). Ancestral divergence of Rickettsia bellii from the spotted fever and typhus groups of Rickettsia and antiquity of the genus Rickettsia. Int J Syst Bacteriol 44, 798-804.

Swofford, D. L. (2000). PAUP ${ }^{*}$ - Phylogenetic Analysis Using Parsimony and other methods (software). Sunderland, MA: Sinauer Associates.

Thompson, J. D., Higgins, D. G. \& Gibson, T. J. (1997). The CLUSTALX-Windows interface: flexible strategies for multiple sequence alignment aided by quality analysis tool. Nucleic Acids Res 25, 4876-4882.

Vitorino, L., Zé-Zé, L., Sousa, A., Bacellar, F. \& Tenreiro, R. (2003). rRNA intergenic spacer regions for phylogenetic analysis of Rickettsia species. Ann N Y Acad Sci 990, 726-733.

Weiss, E. \& Dasch, G. A. (1991). Introduction to the Rickettsiales and other parasitic or mutualistic prokaryotes. In The Prokaryotes, 2nd edn, pp. 2402-2406. Edited by A. Balows, H. G. Trüper, M. Dworking, W. Harder \& K.-H. Schleifer. New York: Springer.

Zhu, Y., Fournier, P.-E., Eremeeva, M. \& Raoult, D. (2005). Proposal to create subspecies of Rickettsia conorii based on multi-locus sequence typing and an emended description of Rickettsia conorii. BMC Microbiol 5, 11 .

Edited by: J. Parkhill 